\title{
MODELO PARA IDENTIFICAÇÃO DA QUALIDADE DE FRAGMENTOS DE VEGETAÇÃO NATIVA: ESTUDO DE CASO DA BACIA DO RIO PIRACICABA /MG
}

\author{
Ewerton Ferreira Cruz \\ Pontifícia Universidade Católica de Minas Gerais (PUC), Instituto de Ciências Humanas, Pós-Graduação em \\ Geografia - Tratamento da Informação Espacial, Belo Horizonte, MG, Brasil \\ ewertonengambiental@yahoo.com.be
}

\begin{abstract}
Alecir Antonio Maciel Moreira
Pontifícia Universidade Católica de Minas Gerais (PUC), Instituto de Ciências Humanas, Pós-Graduação em Geografia - Tratamento da Informação Espacial, Belo Horizonte, MG, Brasil
\end{abstract} alecirmoreira@hotmail.com

\begin{abstract}
RESUMO
A fragmentação de habitats impacta a biodiversidade no sentido de reduzi-los nos níveis de organização estrutural, taxonômica e funcional, bem como genético, específico e ecossistêmico. Apesar de possíveis causas naturais, ela tem sido intensificada pela ação antrópica. Habitats naturais têm sido transformados em mosaicos paisagísticos onde manchas de vegetação nativa se diluem entre áreas modificadas. A fragmentação induz a perda da qualidade do habitat por, entre outros motivos, modificar a sua forma e métrica, potencializando a vulnerabilidade ambiental. Além da perda da qualidade de hábitat, a fragmentação reduz as taxas de migração e/ou recolonização de espécies. Dessa forma, o objetivo desta pesquisa foi elaborar um modelo de mensuração de qualidade de fragmentos de vegetação nativa, baseado em sua métrica. Para atingir os objetivos foram selecionadas as variáveis, tamanho do fragmento, isolamento e índice de circularidade. Para o processamento dos dados, foi utilizado o software ArcGis. O modelo criado foi aplicado à bacia do rio Piracicaba-MG. Observou-se maior concentração de vegetação nativa na região meridional da bacia, onde estão localizadas as nascentes dos principais tributários do rio principal. Cerca de $80 \%$ dos fragmentos de vegetação foram classificados como dotados de qualidade muito alta, ou seja, são mais propícios à conservação.
\end{abstract}

Palavras-chave: Fragmentação. Biogeografia. Métricas da vegetação.

\section{MODEL FOR IDENTIFYING QUALITY OF NATIVE VEGETATION FRAGMENTS: A CASE STUDY OF PIRACICABA RIVER BASIN / MG}

\begin{abstract}
Fragmentation impacts biodiversity in the sense of its reduction on structural, taxonomic and functional levels of organization, as well as genetic, specific and ecosystemic. Despite of possible triggering natural causes, this process is intensifying by means of anthropogenic action. Natural habitats have been transformed into landscape mosaics where native patches of vegetation are diluted within modified areas. Fragmentation induces the loss of habitat quality by means of, among other factors, modifying its metrics and shape, as well as boosting the environmental vulnerability. Besides of loss of habitat quality, fragmentation leads to a decrease in migration and / or recolonization of species rates. Thus, the goal of this research was to create a native vegetation fragment quality measurement model based on its metrics. To reach the goals, size, isolation and circularity index were the selected parameters. In order to obtain the metrics of fragments, ArcGis software was used. The resulting model was applied to Piracicaba river basin (MG). It was observed that major concentrations of natural land cover are located in the south, where are also found the springs of the basin main river tributaries. About $80 \%$ of land cover fragments were considered high quality ones and propitious for conservation finalities.
\end{abstract}

Keywords: Fragmentation. Biogeography. Vegetation metrics.

\section{INTRODUÇÃO}

Muitos são os desafios desencadeados pela crise ambiental contemporânea. Um deles diz respeito ao entendimento do funcionamento dos sistemas ambientais e a sua complexa teia de interações. Incluise aí a dimensão humana do ambiente em seus diferentes matizes culturais. Um segundo desafio diz

$\begin{array}{lllll}\text { Caminhos de Geografia } \quad \text { Uberlândia-MG } & \text { v. 22, n. } 83 & \text { out./2021 } & \text { p. 176-186 } & \text { Página } 176\end{array}$


respeito às técnicas desenvolvidas para avaliação, intervenção e gestão nos (dos) sistemas. Em ambos os casos, o pano de fundo relaciona-se à forma de produção do conhecimento, analítica em essência, em um universo de complexidade. Somam-se tantos outros quanto a intensificação da ocupação humana, mudanças de percepção e acessibilidade seletiva à informação, a multiplicação de impactos e riscos e a própria variabilidade inerente aos sistemas ambientais. Para fins desse trabalho, optou-se por contribuir com o aspecto técnico dessa reflexão e para tanto, a bacia do rio Piracicaba foi eleita como lócus de sua aplicação.

O rio Piracicaba, afluente da margem esquerda do rio Doce, possui um papel importante no contexto socioeconômico do estado de Minas Gerais. Sua bacia, cuja dimensão é de $5.765 \mathrm{~km}^{2}$ e concentra uma população aproximada de 800 mil pessoas. Coberta majoritariamente pelo bioma Mata Atlântica em suas diferentes fitofisionomias, a bacia sofreu forte impacto da ação humana, especialmente em tempos recentes. Incorporada ao centro dinâmico da economia brasileira, a bacia possui forte contribuição no setor de mineração e indústria. Como derivação dessa incorporação, a cobertura vegetal tem sido constantemente suprimida, dando origem a um mosaico paisagístico fragmentado e heterogêneo, com repercussões sobre a biodiversidade, topo e mesoclimas e o comportamento hidrológico da bacia. Por esse motivo o objetivo desse trabalho foi avaliar o estado de conservação da bacia, através da análise da qualidade dos seus fragmentos de sua vegetação nativa. Optou-se pela utilização de geotecnologias como forma de aproximação da dinâmica ambiental recente. A meta foi construir um modelo de análise simples e aplicável que poderá contribuir para a criação de procedimentos para viabilizar a gestão e conservação de fragmentos de vegetação que possuem capacidade de, perpetuação e expansão, transformando e melhorando a qualidade do mosaico paisagístico.

A principal ameaça à perda ou diminuição da biodiversidade é a fragmentação de habitats (SAUNDERS, HOBBS e MARGULES, 1991). A fragmentação impacta diretamente a riqueza de espécies (variedade de vida em todos os níveis de organização estrutural, taxonômica e funcional; diversidade de gene (alelos), espécies e ecossistemas) (SARKAR, 2012).

A fragmentação pode ser derivada de fenômenos naturais, porém, vem sendo intensificada pela ação antrópica. "Muitos habitats naturais que eram quase contínuos uma ou poucas gerações atrás, foram transformados em uma paisagem em mosaico, formado por manchas isoladas do habitat original (ilhas de habitat) circundadas por áreas transformadas de várias formas" (FERNANDEZ, 1997 apud PAGLIA, FERNANDEZ e MARCO JR., 2006, p. 281). Essas modificações tendem a reduzir o fluxo gênico, bem como diminuir os recursos disponíveis para espécies especialistas.

A fragmentação de habitats exerce influências diferenciadas para cada tipo de espécie, de forma a impactar algumas, mais do que outras. De acordo com Losos e Ricklefs (2010), algumas espécies diminuem o número de indivíduos acentuadamente ou desaparecem. Outras permanecem mais ou menos estáveis enquanto um terceiro grupo pode aumentar o número de indivíduos, por vezes drasticamente. As consequências da fragmentação são diversas, porém, duas delas são imediatas: a subdivisão do habitat anteriormente contínuo e a perda de área (PAGLIA, FERNANDEZ e MARCO JR., 2006).

Um suporte teórico importante para a definição da qualidade dos fragmentos de vegetação é oriundo da Teoria da Biogeografia de Ilhas - TBI, presente na ciência denominada Biogeografia. A Biogeografia é uma ciência que envolve duas áreas principais de conhecimento: a Biologia e a Geografia. A Biogeografia pode ser definida como a ciência que tenta documentar e compreender os padrões espaciais da biodiversidade através do estudo das distribuições de organismos, tanto do passado como do presente, e de padrões relacionados à variação da quantidade e de tipos de seres vivos no planeta (BROWN e LOMOLINO, 1998). Deve-se ressaltar que, embora a definição possa parecer, de certa forma, simplista, os fatores que influenciam nas distribuições dos organismos são complexos, pois, eles são, muitas vezes, inter-relacionados além de envolver várias abordagens metodológicas de várias disciplinas científicas distintas (FIGUEIREDO, SILVA e SOUZA, 2006).

A Teoria da Biogeografia de llhas - TBI presente nessa área de conhecimento discorre e explica os fenômenos biológicos que nelas ocorrem. Apesar de ter sido desenvolvida para estudar as ilhas geográficas, a sua aplicação tem sido de valia para compreensão da dinâmica ambiental estabelecida em ilhas biogeográficas, geradas por processo de fragmentação (PAGLIA, FERNANDEZ e MARCO JR., 2006). As ilhas verdadeiras têm sua biota influenciada por diversos fatores dentre os quais, seu tamanho e grau de isolamento.

As ilhas podem ser definidas, em termos biogeográficos, como "porções de habitat cercados por um habitat diferente e que represente uma barreira, mesmo que parcial, para a dispersão do táxon em

$\begin{array}{lllll}\text { Caminhos de Geografia } & \text { Uberlândia-MG } & \text { v. 22, n. 83 } & \text { out./2021 } & \text { p. 176-186 }\end{array}$


questão" (PAGLIA, FERNANDEZ, MARCO JR., 2006, p. 284). As ilhas englobam não somente as ilhas geográficas, aquelas cercadas por água, mas também aquelas geradas por processo de fragmentação (fragmentos de vegetação, por exemplo). As ilhas são habitats isolados, de tamanho relativamente pequeno, contendo diversidade limitada de habitats e recursos (BROWN e LOMOLINO, 1998).

$\mathrm{O}$ tamanho e o isolamento das ilhas são diretamente proporcionais à diversidade de espécies encontradas nessas áreas. As ilhas de maiores extensões possuem, além de maior quantidade de indivíduos, maior número de espécies. Esse fenômeno ocorre porque quanto maior a área, maior a probabilidade de ocorrência de habitats distintos, e, como apontado por Brown e Lomolino (1998), há maior chance de haver espécies especializadas que são restritas a cada um desses diferentes habitats. De forma análoga, quanto menos isoladas são as ilhas, maior a probabilidade de ocorrência de maior número de indivíduos e espécies. O isolamento é um fator que influência na variabilidade genética dos indivíduos, pois, se houver um isolamento dificultará a troca de material genético entre os indivíduos, podendo diminuir a capacidade de adaptação dos mesmos. Assim sendo, o isolamento e o tamanho das ilhas influenciam diretamente na taxa de extinção de espécies.

Além do tamanho e do isolamento, os fragmentos de vegetação (ilhas) também são influenciados por fatores tais como: seu entorno, o efeito de borda e a história da fragmentação (OLIFIERS e CERQUEIRA, 2006). Dessa forma, fatores mensuráveis podem ser indicadores de qualidade de fragmentos de vegetação para fins de conservação e preservação da biodiversidade. Eles podem ser obtidos através do uso de Sistemas de Informação Geográfica - SIGs, o que viabiliza a criação de rotinas e modelos com vistas a sua aplicação.

Deve-se destacar que ferramentas computacionais são de grande valia para o estudo de paisagens, especialmente em grandes espaços territoriais. Os SIGs possuem as principais ferramentas para estudos ambientais e, por conseguinte, análise de fragmentos de vegetação e suas métricas. O SIG

é composto por um conjunto de ferramentas computacionais (equipamentos e programas), que permite não só o armazenamento, processamento e gerenciamento de dados espaciais, mas também diversas formas de consulta, análise, visualização e produção de resultados tabulares, gráficos e digitais (BECKER, 2005, p. 93).

Ele é utilizado para análise de objetos de natureza espacial quando é de extrema importância a localização geográfica de um determinado fenômeno em relação a outros (BECKER, 2005). De forma sucinta, o SIG possui funções e fórmulas estatísticas necessárias para o tratamento de dados e de imagens digitais.

Dessa forma, esta pesquisa teve como finalidade a proposição de um modelo para identificar a qualidade dos fragmentos de vegetação nativa, com base em suas métricas. A partir da sua criação, foi possível aplicá-lo à análise dos fragmentos de vegetação na bacia do rio Piracicaba-MG. A escolha da bacia para fins de teste do modelo deveu-se ao fato de ter sido impactada pela ocorrência do desastre ambiental da ruptura da barragem de Fundão em Mariana-MG.

Nessa perspectiva, o estudo poderá contribuir para a análise ambiental através da identificação de áreas para fins de recuperação e/ou prioritárias à conservação. Ele poderá, ainda, contribuir diretamente e indiretamente para a proteção dos recursos hídricos locais. Através da aplicação do modelo gerado na bacia do rio Piracicaba será possível, através dos mecanismos de gestão de nível municipal e estadual, subsidiar o melhor uso do território da bacia. Seu principal desafio foi abordar as métricas dos fragmentos de vegetação através da articulação de variáveis por meio da análise espacial.

\section{PROCEDIMENTOS METODOLÓGICOS}

Diversos métodos têm sido utilizados para identificação de áreas com altos valores para conservação. Muitos partem de levantamentos sistemáticos ou rápidos da diversidade biológica, em áreas específicas. Em outras palavras, partem da constatação da riqueza de espécies para o apontamento da área. Esses métodos são essenciais e poderosos, mas têm como contrapartida uma demanda de tempo e recursos nem sempre disponíveis. Eles são largamente estimulados por imposições legais para licenciamento de empreendimentos. Apesar de sua qualidade, seu uso pode gerar padrões espaciais de diversidade não correspondentes à riqueza propriamente dita, mas, antes, refletirem a espacialização de interesses econômicos, já que nem todo espaço é sistematicamente avaliado.

Este fenômeno foi observado por Moreira (2015) em análise de áreas prioritárias para conservação na bacia do rio Paraopeba (sub-bacia do Rio São Francisco) localizada na região central do Estado Minas

$\begin{array}{lllll}\text { Caminhos de Geografia } \quad \text { Uberlândia-MG } & \text { v. 22, n. 83 } & \text { out./2021 } & \text { p. 176-186 Página } 178\end{array}$


Gerais, englobando total ou parcialmente 48 municípios com área de $12.054,25 \mathrm{~km}^{2}$. Uma solução possível para o problema seria partir da análise de feições espaciais que apontem para a integridade da qualidade dos ambientes. Nessa perspectiva, uma das possibilidades consiste na análise morfológica de remanescentes de vegetação nativa. Ela subentende a ideia de qualidade ambiental e insere-se no âmbito da ecologia da paisagem. Trata-se de um método simples e pouco oneroso de identificação de áreas de interesse. A partir daí podem-se suceder os levantamentos sistemáticos. Ele não esgota as possibilidades de identificação de riqueza biológica, mas pode contribuir para tornar mais objetivos e rápidos os diagnósticos ambientais.

A qualidade dos fragmentos de vegetação, segundo Freitas (2012), é influenciada por perturbações que podem vir da proximidade dos fragmentos com o uso e ocupação do solo nas terras e nas bordas, a intensidade e o tamanho dos fragmentos e a cobertura vegetal nativa. Neste estudo, o modelo foi criado considerando as características: tamanho, índice de circularidade e menor distância do fragmento mais próximo (Figura 1).

Posteriormente, para a aplicação do modelo na bacia do rio Piracicaba, foram utilizados dados vetoriais referentes aos remanescentes de vegetação do mapeamento florestal do Estado de Minas Gerais realizado pelo IEF em 2009 cujo intuito foi a identificação dos remanescentes florestais nativos por meio do processamento digital de imagens de satélite e sensoriamento remoto. As métricas dos fragmentos foram obtidas através do processamento dos dados vetoriais através do software ArcGis versão 10.3 da empresa ESRI no laboratório de Cartografia do Programa de Pós-graduação em Geografia Tratamento da Informação Espacial da Pontifícia Universidade Católica de Minas Gerais.

Figura 1 - Fluxograma do método utilizado.

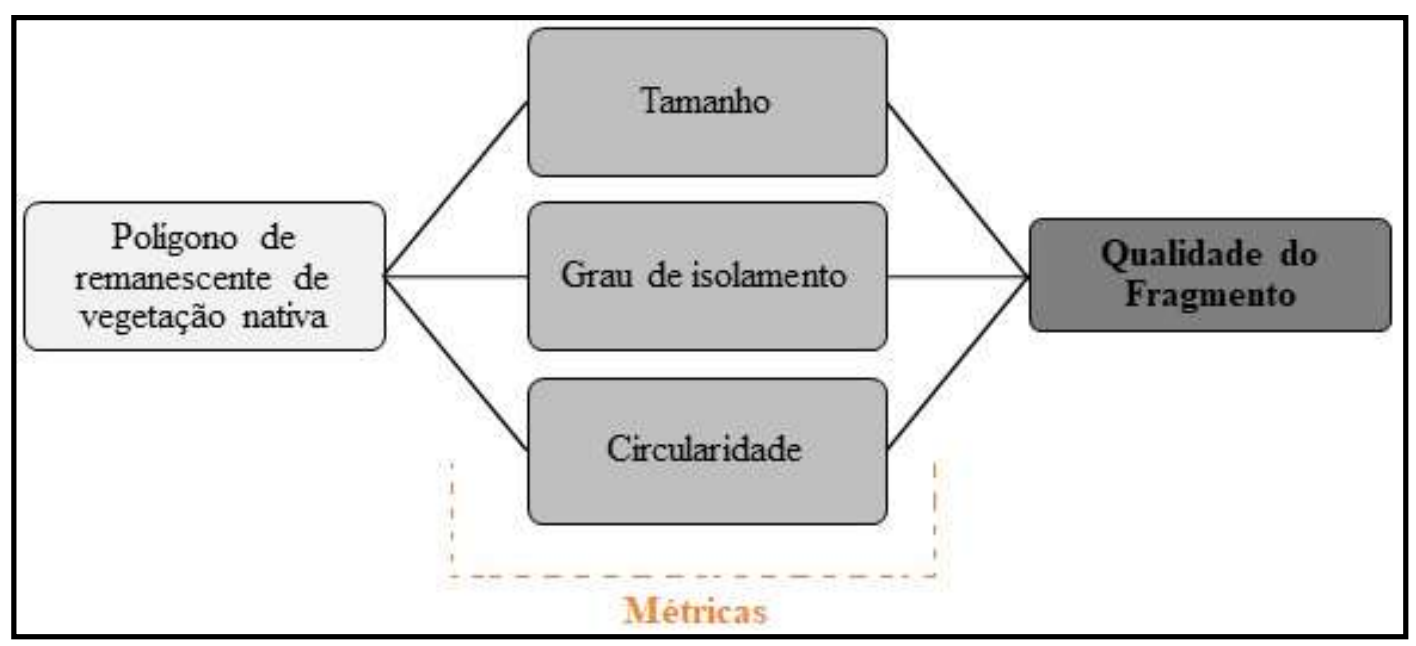

Fonte - Os autores.

O efeito do tamanho dos remanescentes foi extraído da TBI da relação espécie-área. Segundo esta, a quantidade de espécies é proporcional ao tamanho do fragmento. Outros fatores são influenciados pelo tamanho do fragmento. Pequenos fragmentos são mais suscetíveis à ocorrência de eventos estocásticos do que aqueles maiores.

Outro ponto importante é a quantidade de habitats presentes em fragmentos menores. Segundo Williams (1964), pequenas áreas tendem a possuir um número menor de diferentes habitats, o que, potencialmente, reduz o número de espécies associadas a esses. O tamanho dos fragmentos influencia diretamente e indiretamente a riqueza de espécies.

Quando o parâmetro tamanho se soma à distância do fragmento em relação a outros, maior é seu grau de perturbação, portanto, sua qualidade para conservação e preservação é baixa (BROWN e LOMOLINO, 1998). De forma analogamente inversa, quanto maior for o fragmento, menor, espera-se, seja o seu grau de perturbação apresentando uma maior qualidade ambiental. A classificação da qualidade dos fragmentos em relação à sua área foi baseada em Fengler et al. (2015), conforme o quadro 1.

$\begin{array}{lllll}\text { Caminhos de Geografia } & \text { Uberlândia-MG } & \text { v. 22, n. } 83 & \text { out./2021 } & \text { p. 176-186 }\end{array}$


Quadro 1 - Pesos atribuídos para o tamanho do fragmento.

\begin{tabular}{|l|l|l|}
\hline \multicolumn{1}{|c|}{ Área (ha) } & \multicolumn{1}{|c|}{ Qualidade } & Peso \\
\hline$>0,5$ & Muito baixa & 1,0 \\
\hline $0,5-1,0$ & Baixa & 1,5 \\
\hline $1,0-5,0$ & Média & 2,0 \\
\hline $5,0-20,0$ & Alta & 2,5 \\
\hline$>20$ & Muito alta & 3,0 \\
\hline
\end{tabular}

Fonte - FENGLER et al. (2015). Adaptado pelos autores.

O grau de isolamento, segundo a TBI, afeta proporcionalmente o número de espécies dos fragmentos. Isso ocorre porque fragmentos mais isolados tendem a ter reduzida a taxa de colonização e/ou recolonização de espécies advindas de outras áreas próximas (OLIFIERS e CERQUEIRA, 2006). Em outras palavras, "a dificuldade de movimentação de organismos entre fragmentos é proporcional ao grau de isolamento destes" (ALMEIDA, 2008, p. 27).

Outro fator influenciado pelo isolamento é a aleatoriedade genética, potencializando a extinção de espécies. Tal como o tamanho, o grau de isolamento influencia diretamente e indiretamente a biodiversidade dos fragmentos. O grau de isolamento é verificado através da métrica do vizinho mais próximo. Dessa maneira, a qualidade dos fragmentos em relação ao isolamento foi determinada conforme o modelo proposto por Almeida (2008) como demonstrado no quadro 2.

Quadro 2 - Pesos atribuídos para a distância entre o fragmento mais próximo.

\begin{tabular}{|l|l|l|}
\hline Distância $(\mathbf{m})$ & Qualidade & Peso \\
\hline$>400$ & Muito baixa & 1,0 \\
\hline $200-400$ & Baixa & 1,5 \\
\hline $120-200$ & Média & 2,0 \\
\hline $60-120$ & Alta & 2,5 \\
\hline$<60$ & Muito alta & 3,0 \\
\hline
\end{tabular}

Fonte - ALMEIDA (2008). Adaptado pelos autores.

Alterações ambientais no entorno de fragmentos impactam o(s) ecossistema(s) ali presente(s), de modo a criar um meio perturbado (FREITAS, 2012). Essas perturbações podem ser denominadas "efeito de borda". Este, por seu turno, está relacionado à forma do fragmento. O efeito de borda pode ser conceituado como o resultado do contato entre dois ambientes próximos que estão separados por uma transição brusca (MURCIA, 1995). Em outras palavras, o efeito de borda está pautado em mudanças abióticas e bióticas que ocorrem no limite do fragmento, podendo adentrá-lo em diferentes direções e distâncias.

Mudanças abióticas são consequências do ecótono (áreas de transição ambiental onde há contato entre as diferentes comunidades ecológicas) (LOVEJOY et al., 1986). Segundo Murcia (1995), a heterogeneidade do ecótono ocasiona variações microclimáticas que modificam a estrutura da vegetação, bem como influencia as comunidades animais do fragmento. Alguns exemplos do que o

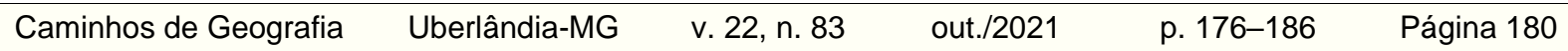


efeito de borda pode causar são: alterações na temperatura, umidade, incidência de ventos, quedas de árvores, formação de clareiras, aumento na abundância de cipós, lianas e plantas de sucessão secundária (OLIFIERS, CERQUEIRA, 2006).

Fragmentos pequenos estão mais expostos ao efeito de borda, visto que a distância entre esta e o seu centroide é menor. Por esse motivo, fragmentos de maior porte são prioritários para a conservação, pois alterações físicas e microclimáticas em suas bordas são menores (SCARANO, 2006). Pode-se assumir que, quanto maior for a circularidade do fragmento, menos intensos serão os efeitos de borda e melhor a conservação do ecossistema local, possibilitando uma melhor manutenção do meio. O índice de circularidade é função da área e do perímetro do polígono. Para a obtenção dessa variável foi utilizada a equação (1) proposta por Silva Neto et al. (2013):

$$
I C=\frac{12,54 x A}{P^{2}}
$$

Onde:

A = Área;

$\mathrm{P}=$ Perímetro;

IC = Índice de circularidade.

Por fim, a qualidade dos fragmentos em relação à sua circularidade foi analisada conforme a classificação proposta por Fengler et al. (2015) atribuindo-se pesos conforme disposto no quadro 3.

Quadro 3 - Pesos atribuídos para o índice de circularidade do fragmento.

\begin{tabular}{|c|l|l|}
\hline \multicolumn{1}{|c|}{ IC } & \multicolumn{1}{|c|}{ Qualidade } & Peso \\
\hline $0,0-0,2$ & Muito baixa & 1,0 \\
\hline $0,2-0,4$ & Baixa & 1,5 \\
\hline $0,4-0,6$ & Média & 2,0 \\
\hline $0,6-0,8$ & Alta & 2,5 \\
\hline $0,8-1,0$ & Muito alta & 3,0 \\
\hline
\end{tabular}

Fonte - Os autores.

Ao final foi realizada uma média aritmética dos valores obtidos em cada varável (área, distância e IC), conforme a equação (2) abaixo, e atribuídos os pesos para as unidades prioritárias de conservação, (Quadro 4).

$$
F=\frac{T+D+C}{3}
$$

Onde:

$\mathrm{F}=$ Qualidade do fragmento;

$\mathrm{T}=$ Tamanho do fragmento;

$\mathrm{D}$ = Distância entre o fragmento mais próximo;

$\mathrm{C}=$ Circularidade do fragmento. 
Quadro 4 - Pesos atribuídos para a classificação da qualidade do fragmento.

\begin{tabular}{|l|l|}
\hline \multicolumn{1}{|c|}{ Qualidade } & \multicolumn{1}{c|}{ Média } \\
\hline Muito baixa & 1,0 \\
\hline Baixa & 1,5 \\
\hline Média & 2,0 \\
\hline Alta & 2,5 \\
\hline Muito alta & 3,0 \\
\hline
\end{tabular}

Fonte - Os autores.

\section{O PROCESSO DE USO E OCUPAÇÃO DA BACIA DO RIO PIRACICABA/MG}

A Bacia Hidrográfica do Rio Piracicaba - BHRP, localizada no Estado de Minas Gerais, possui uma área de aproximadamente $5.706 \mathrm{~km}^{2}$ (Figura 2). Próximos às nascentes do rio Piracicaba, localizadas no municipio de Ouro Preto, foram encontrados os maiores remanescentes de vegetaçã̃o nativa da bacia, o que é de fundamental importância para a manutenção da qualidade da água. É importante destacar que junto à foz do rio Piracicaba no Rio Doce, entre os municípios de Ipatinga (MG) e Timóteo (MG), também foi localizada uma expressiva área vegetada.

Percorrido por bandeirantes desde o início da conquista do território mineiro, o Piracicaba apresenta sinais evidentes de degradação. Atualmente, em sua bacia estão instaladas diversas mineradoras, siderúrgicas e é largamente difundida a silvicultura do eucalipto. Todas essas atividades industriais têm grande potencial de degradação para a vegetação nativa remanescente e a qualidade do ambiente. Ressalta-se que a ocupação da bacia está diretamente relacionada à exploração de recursos naturais (CRUZ, 2009).

Figura 2 - Mapa de localização da bacia do Rio Piracicaba.

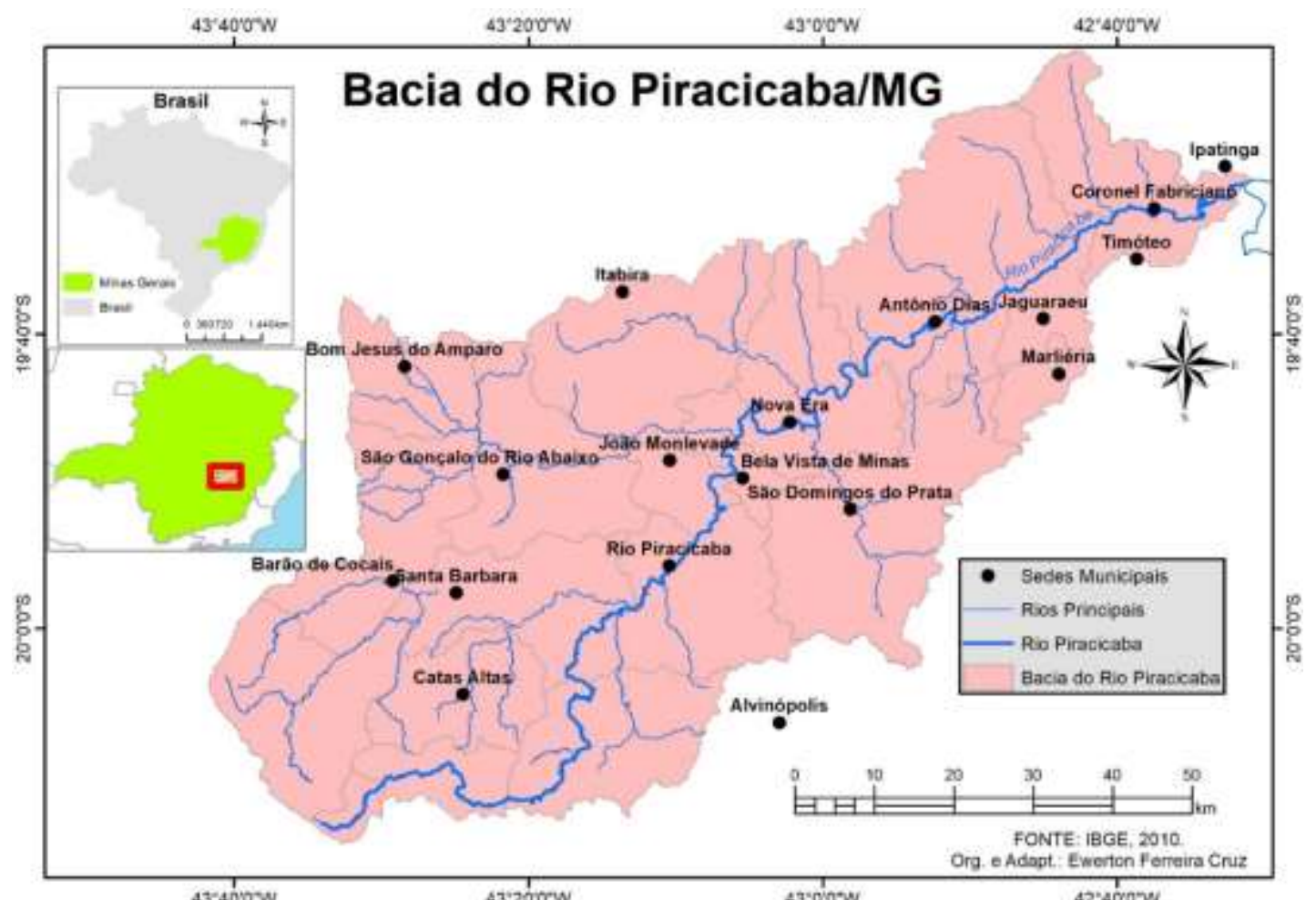

Fonte - Os autores.

Caminhos de Geografia Uberlândia-MG $\quad$ v. 22, n. $83 \quad$ out./2021

p. $176-186$

Página 182 
De acordo com Paula (1997), a ocupação da bacia teve como propulsor o início da extração de ouro no final século XVII nos municípios de Ouro Preto e Mariana, dois dos mais antigos e importantes da história do Brasil. Com a busca intensa desse mineral em diversas áreas próximas a esses municípios, diversos arraiais foram fundados, as quais posteriormente originaram as cidades de Antônio Dias, Catas Altas, Itabira, Santa Bárbara e Nova Era. Esse processo de ocupação fez com que diversas áreas de vegetação nativa fossem suprimidas, porém, em pequena escala.

Posteriormente, no século XX, houve a construção da Estrada de Ferro Vitória-Minas, fazendo com que novas ocupações surgissem, bem como a instalação de novas industrias. A descoberta de novas jazidas de minério de ferro atraiu empresas do ramo minerário para a região, realimentando o processo de ocupação. Indústrias de beneficiamento de minério, as siderúrgicas (BRAGA, 1998), foram para ali atraídas. Logo, induziu-se a necessidade de produção de carvão vegetal, que serve como combustível no processo de tratamento do minério de ferro. Favorecidas por incentivos fiscais e políticas públicas, empresas começaram a investir no reflorestamento através da monocultura de eucalipto. Este processo histórico induziu, por seu turno, a uma contínua ação de supressão e de fragmentação da cobertura vegetal, com impactos diretos e indiretos sobre a qualidade ambiental.

Para tornar ainda mais complexa a situação da bacia, no ano de 2015, a bacia do rio Doce foi fortemente impactada por um desastre ambiental decorrente da atividade minerária de uma empresa de grande porte, que afetou, a jusante, larga porção do território mineiro: o desastre do rompimento da barragem de Fundão em Mariana (MG). Entre os impactos do desastre incluem-se a morte de milhares de peixes e animais, a poluição de águas e o assoreamento de cursos d'água, a supressão de vegetação ciliar e a contaminação de solos.

Apesar da maior parte das atividades industriais da região estar voltada para a mineração, a região possui também uma forte concentração de pecuária diversificada, bem como complexos agroindustriais (CRUZ, 2009). Por causa dessa grande diversidade de atividades econômicas, a bacia do Rio Piracicaba possui problemas ambientais variados, que ainda são intensificados pelo processo de urbanização (CRUZ, 2009).

Além dos problemas ambientais oriundos, de forma direta, das atividades minerárias, a instalação de novas industrias ocasionou o crescimento populacional da região durante todo o seu período histórico de formação. De acordo com Plano de Ação de Recursos Hídricos da Unidade de Planejamento e Gestão dos Recursos Hídricos Piracicaba (IGAM, 2010), a bacia do Rio Piracicaba foi a que apresentou o maior ritmo de crescimento populacional da bacia do Rio Doce, apresentando um índice médio de crescimento de 1,20\% ao ano entre os anos de 2000 e 2007. Esse crescimento populacional implica diretamente a criação de equipamentos de infraestrutura de saneamento.

Este processo histórico de uso e ocupação do território induziu uma contínua ação de supressão e de fragmentação da cobertura vegetal, com impactos diretos e indiretos sobre a qualidade ambiental. "No sudeste do Brasil [...] a fragmentação já atinge um estágio muito avançado e a preservação das áreas remanescentes da Mata Atlântica nesta região foi apontada como um dos maiores problemas de conservação do País" (PAGLIA, FERNANDEZ, MARCO JR., 2006, p. 281). Para além da perda de cobertura vegetal e da fragmentação de habitats, o processo de produção do espaço regional (brasileiro, em geral) condicionou a existência de um conjunto de problemas socioambientais de grande monta.

\section{A QUALIDADE DOS FRAGMENTOS DA BACIA DO RIO PIRACICABA/MG}

As análises revelaram que a distribuição da vegetação nativa da bacia do Rio Piracicaba está mais concentrada em sua porção meridional. Aí e no centro-norte estão localizadas as nascentes dos principais tributários do rio principal. As regiões central e noroeste possuem menor número de fragmentos de vegetação e, consequentemente, baixa conectividade (Figura 3).

A partir do cálculo métrico observou-se (Figura 3) que a maior parte dos fragmentos de vegetação (cerca de $80 \%$ ) pode ser classificada como dotada de qualidade muito alta, ou seja, são mais propícios a serem conservados. A concentração e a extensão dos fragmentos aí localizados corroboram o método utilizado.

Verificou-se ainda a existência de uma concentração de fragmentos classificados como de média qualidade na região centro-leste. Os fragmentos com qualidade ruim estão majoritariamente

$\begin{array}{lllll}\text { Caminhos de Geografia } \quad \text { Uberlândia-MG } & \text { v. 22, n. 83 } & \text { out./2021 } & \text { p. 176-186 } & \text { Página } 183\end{array}$


distribuídos ao centro, centro-oeste e norte da bacia. Os fragmentos classificados como de qualidade muito ruim inexistem na bacia (Figura 3).

Figura 3 - Mapa do Índice de Qualidade dos Fragmentos de Vegetação da bacia do Rio Piracicaba.

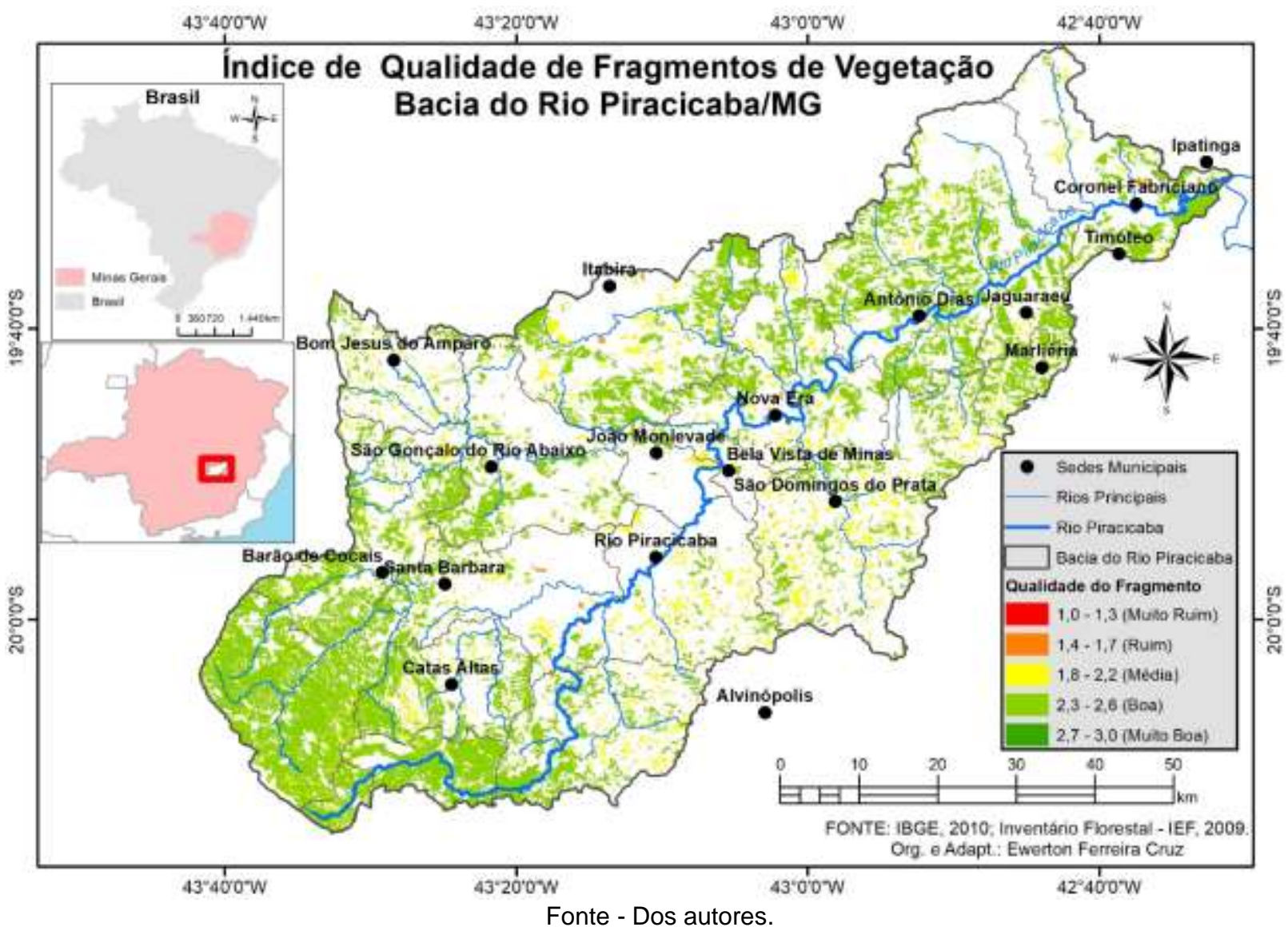

É importante ressaltar que, as áreas não classificadas, são aquelas que não possuem fragmentos de acordo com o mapeamento realizado pelo IEF em 2009. Essas áreas, provavelmente, são áreas urbanas, agrícolas ou pastagens, não interessantes para a conservação.

\section{CONSIDERAÇÕES FINAIS}

A criação de modelos para a valoração dos remanescentes de vegetação nativa é de fundamental importância para a gestão territorial e, consequentemente, manutenção ou melhoria da qualidade ambiental. Rotinas relativamente simples de uso de SIGs e parâmetros notadamente relevantes de identificação de áreas de boa qualidade ambiental podem subsidiar a tomada de decisão para delimitação de áreas prioritárias para conservação.

Através da atribuição de pesos para valorar a qualidade de fragmentos de vegetação pôde-se perceber que existem diversas possibilidades para a criação de modelos. Há muitas características/parâmetros que devem ser consideradas na valoração de um determinado fragmento. Alguns desses parâmetros não podem ser quantificados, o que dificulta o seu mapeamento através da álgebra de mapas. Neste trabalho optou-se pela classificação dos fragmentos de vegetação tendo por base as suas métricas. Cabe salientar que, este modelo deve ser usado como uma medida complementar à caracterização qualitativa dos fragmentos bem como ao uso outros modelos relacionados ao meio físico da área a ser avaliada.

Finalmente, quanto à sua aplicação na bacia do rio Piracicaba, ele apontou a existência de áreas que possuem maiores concentrações fragmentos, bem como a baixa quantidade de fragmentos que possuem qualidade ruim. Infere-se que, a despeito de uma ocupação secular e do modo de produção

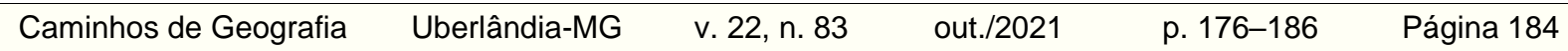


do espaço vigente, a maior parte dos fragmentos existentes da bacia tem potencial considerável para exibição de uma rica biodiversidade.

\section{AGRADECIMENTOS}

À CAPES pela concessão de bolsa de pesquisa ao primeiro autor pelo período compreendido entre março/2016 e fevereiro/2018.

\section{REFERÊNCIAS}

ALMEIDA, Cristina Guilherme de. Análise especial dos fragmentos florestais na área no Parque Nacional dos Campos Gerais, Paraná. Dissertação de Mestrado. UEPG. Programa de Pósgraduação em Geografia. Ponta Grossa: 2008. 72 p.

BECKER, Fernando Gertum. Aplicações de Sistemas de Informação Geográfica em Ecologia e Manejo de Bacias Hidrográficas. In: SCHIAVETTI, Alexandre; CAMARGO, Antonio F. M. Conceitos de Bacias Hidrográficas: Teorias e Aplicações. Ilhéus: Editus, 2005. 91-110.

BRAGA, T. Conflito Sócio-Ambiental e Constituição de Agentes Sociais Ambientalistas: um estudo sobre as cidades industriais da bacia do Rio Piracicaba (MG). Belo Horizonte, 1998. Disponível em: <http://ebookbrowse.com/r/rio-piracicaba> Acesso em: 9 nov. de 2017.

BROWN, James H.; LOMOLINO, Mark, V. Biogeography. Second edition. Massachusetts: Sinauer Associates, 1998. $691 \mathrm{p}$.

CRUZ, Lucas Paiva. Avaliação do Comitê de Bacia Hidrográfica do Rio Piracicaba. Dissertação. Programa de Pós-Graduação em Engenharia Ambiental, Universidade Federal de Ouro Preto. 2009. $139 \mathrm{p}$.

FENGLER, Felipe H.; MORAES, Jener F. L. de; RIBEIRO, Admilson I.; PECHE FILHO, Afonso; STORINO, Moisés; MEDEIROS, Gerson A. de. Qualidade ambiental dos fragmentos florestais na Bacia Hidrográfica do Rio Jundiaí-Mirim entre 1972 e 2013. Revista Brasileira de Engenharia Agrícola e Ambiental. Campina Grande: 2015, v.19, n.4, p.402-408. https://doi.org/10.1590/18071929/agriambi.v19n4p402-408

FIGUEIREDO, Wilsea Maria Batista; SILVA, José Maria Cardoso da; SOUZA, Manuella Andrade de. Biogeografia e a conservação da biodiversidade. In: ROCHA, Carlos Frederico Duarte; BERGALLO, Helena Godoy; SLUYS, Monique Van; ALVES, Maria Alice Santos (Org.). Biologia da conservação: essências. São Carlos: RiMa Editora, 2006. p. 135-156.

FREITAS, Eduardo Pretto. Análise integrada do mapa de uso e ocupação das terras da microbacia do Rio Jundiaí-Mirim para fins de gestão ambiental. (Dissertação Mestrado). Campinas: Instituto Agronômico de Campinas, 2012. 110p.

INSTITUTO MINEIRO DE GESTÃO DAS ÁGUAS - IGAM. Plano de Ação de Recursos Hídricos da Unidade de Planejamento e Gestão dos Recursos Hídricos Piracicaba- PARH Piracicaba. Consórcio ECOPLAN Engenharia e LUME Estratégia Ambiental. Maio de 2010. 100 p. Disponível em: $<$ http://www.cbhdoce.org.br/wp-content/uploads/2015/01/PARH Piracicaba.pdf $>$. Acesso em: 5 jan. 2017.

LOSOS, Jonathan B.; RICKLEFS, Robert E. The Theory of Island Biogeography Revised. New Jersey: Princeton University Press, 2010. 476 p. https://doi.org/10.1515/9781400831920

LOVEJOY, T. E.; BIERREGAARD JR., R. O.; RYLANDS, A. B.; MALCOLM, J. R.; QUINTELA, C. E.; HARPER, L. H.; BROWN JR., K. S.; POWELL, A. H.; PPOWELL, G. V. N.; SCHUBART, H. O. R.; HAYS, M. B. Edge and other effects of isolation on Amazon forest fragments. 1986, p. 257-285. In: SOULÉ, M. E. (editor). Conservation biology: the science of scarcity and diversity. Sunderland: Sinauer Associates, 1986.

MOREIRA, Alecir Antonio Maciel. Desafios à conservação na Bacia do Paraobeba-MG: identificando valores. Tese de doutorado. Pontifícia Universidade Católica de Minas Gerais. Programa de Pós-graduação em Geografia - Tratamento da Informação Espacial. Belo Horizonte, 2015, $287 \mathrm{p}$.

$\begin{array}{lllll}\text { Caminhos de Geografia } & \text { Uberlândia-MG } & \text { v. 22, n. } 83 & \text { out./2021 } & \text { p. 176-186 }\end{array}$


MURCIA, C. Edge effects in fragmented forests: implications for conservation. Trends in Ecology and Evolution, 1995, vol. 10. p. 58-62. https://doi.org/10.1016/S0169-5347(00)88977-6

OLIFIERS, Natalie; CERQUEIRA, Rui. Fragmentação de habitat: efeitos históricos e ecológicos. In: ROCHA, C. F. D. et al. (Org.). Biologia da conservação: essências. São Carlos: RiMa Editora, 2006. p. 261-279.

PAGLIA, A. P.; FERNANDEZ, F. A. S.; MARCO JR., P. de. Efeitos da fragmentação de habitats: quantas espécies, quantas populações, quantos indivíduos, e serão eles suficientes? In: ROCHA, C. F. D. et al. (Org.). Biologia da conservação: essências. São Carlos: RiMa Editora, 2006. p. 261279.

PAULA, J.A. Biodiversidade, população e economia: uma região de mata atlântica. Belo Horizonte: UFMG/Cedeplar; ECMVC; PADCT/CIAMB , 1997.

SARKAR, Sahotra. Environmental Philosophy: From Theory to Practice. Malden: Wiley-Blackwell, 2012. $226 \mathrm{p}$.

SAUNDERS, D. A.; HOBBS, R. J.; MARGULES, C. R. Biological consequences of ecosystem fragmentation: a review. Conservation Biology, 1991, volume 5. p. 18-32. https://doi.org/10.1111/j.1523-1739.1991.tb00384.x

SCARANO, Fabio Rubio. Prioridades para conservação: a linha tênue que separa teorias e dogmas. In: ROCHA, C. F. D. et al. (Org.). Biologia da conservação: essências. São Carlos: RiMa Editora, 2006. p. 23-39.

SILVA NETO, A. F. da; et al. Geotecnologias para a caracterização morfométrica de bacias hidrográficas. Anais XVI Simpósio Brasileiro de Sensoriamento Remoto - SBSR, Foz do Iguaçu: 2013, INPE. p. 5473-5490.

WILLIAMS, C. B. Patterns in the balance of nature. London: Academic Press, 1964.

Recebido em: 23/07/2020

Aceito para publicação em: 14/03/2021 